\title{
Correction to: A composite oral hygiene score and the risk of oral cancer and its subtypes: a large-scale propensity score-based study
}

\author{
Qingrong Deng ${ }^{1,2} \cdot$ Lingjun Yan $^{1,2}$. Junyu Lin ${ }^{3} \cdot$ Zhaocheng Zhuang $^{1,2} \cdot$ Yihong Hong $^{1,2}$. Chanchan $\mathrm{Hu}^{1,2}$. \\ Lisong Lin ${ }^{4} \cdot{\text { Lizhen } \mathrm{Pan}^{4} \cdot \text { Bin Shi }^{4} \text {. Jing Wang }}^{5} \cdot$ Fengqiong Liu ${ }^{1,2} \cdot$ Lin Cai $^{1,2} \cdot$ Baochang He $^{1,2} \cdot$ Yu Qiu ${ }^{4} \cdot$ Fa Chen $^{1,2}$
}

Published online: 25 January 2022

(c) Springer-Verlag GmbH Germany, part of Springer Nature 2021

\section{Correction to: Clinical Oral Investigations https://doi.org/10.1007/s00784-021-04209-8}

The online version of the article contained a mistake in the name of the first corresponding author. The correct name is $\mathrm{Fa}$ Chen. There is also a typesetting error in the order of the authors' names.

The original article has been corrected.

Publisher's note Springer Nature remains neutral with regard to jurisdictional claims in published maps and institutional affiliations.

The original article can be found online at https://doi.org/10.1007/ s00784-021-04209-8.

Fa Chen

chenfa@fjmu.edu.cn

$\triangle$ Yu Qiu

dr_qiuyu88@163.com

1 Department of Epidemiology and Health Statistics, School of Public Health, Fujian Medical University, 1 Xueyuan Road, FuzhouFujian 350108, China

2 Key Laboratory of Ministry of Education for Gastrointestinal Cancer, Fujian Medical University, Fuzhou, China

3 Department of Thyroid and Breast Surgery, The First Affiliated Hospital of Fujian Medical University, Fuzhou, China

4 Department of Oral and Maxillofacial Surgery, the First Affiliated Hospital of Fujian Medical University, 88 Jiaotong Road, Fuzhou 350004, China

5 Laboratory Center, School of Public Health, The Major Subject of Environment and Health of Fujian Key Universities, Fujian Medical University, Fujian, China 